\title{
Accuracy mechanism of eukaryotic ribosome translocation
}

https://doi.org/10.1038/s41586-021-04131-9

Received: 24 June 2021

Accepted: 13 October 2021

Published online: 1 December 2021

\section{Open access}

Check for updates

\author{
Muminjon Djumagulov ${ }^{1,2}$, Natalia Demeshkina ${ }^{1,3}$, Lasse Jenner', Alexey Rozov ${ }^{1,2}$ \\ Marat Yusupov $^{1 \bowtie}$ \& Gulnara Yusupova ${ }^{1 凶}$
}

Translation of the genetic code into proteins is realized through repetitions of synchronous translocation of messenger RNA (mRNA) and transfer RNAs (tRNA) through the ribosome. In eukaryotes translocation is ensured by elongation factor 2 (eEF2), which catalyses the process and actively contributes to its accuracy ${ }^{1}$. Although numerous studies point to critical roles for both the conserved eukaryotic posttranslational modification diphthamide in EEF2 and tRNA modifications in supporting the accuracy of translocation, detailed molecular mechanisms describing their specific functions are poorly understood. Here we report a high-resolution X-ray structure of the eukaryotic $80 \mathrm{~S}$ ribosome in a translocation-intermediate state containing $\mathrm{mRNA}$, naturally modified eEF 2 and tRNAs. The crystal structure reveals a network of stabilization of codon-anticodon interactions involving diphthamide ${ }^{1}$ and the hypermodified nucleoside wybutosine at position 37 of phenylalanine tRNA, which is also known to enhance translation accuracy ${ }^{2}$. The model demonstrates how the decoding centre releases a codon-anticodon duplex, allowing its movement on the ribosome, and emphasizes the function of eEF2 as a 'pawl' defining the directionality of translocation ${ }^{3}$. This model suggests how eukaryote-specific elements of the $80 \mathrm{~S}$ ribosome, eEF2 and tRNAs undergo large-scale molecular reorganizations to ensure maintenance of the mRNA reading frame during the complex process of translocation.
In eukaryotes, the complex process of translocation is ensured by eEF2, a GTPase that is indispensable for maintaining the correct mRNA reading frame. Many genetic and biochemical studies point to a critical role of the unique eEF2 post-translational modification diphthamide, which is located in domain IV and is conserved among eukaryotes and archaea. Organisms lacking diphthamide have reduced protein synthesis rates and increased occurrence of $(-1)$ frameshifting ${ }^{1,4}$. Diphthamide is a target of several virulent toxins that inactivate eEF 2 by ADP ribosylation and cause lethal effects ${ }^{5}$.

The current structural knowledge about dynamics of the eukaryotic elongation cycle has been provided by low-to-intermediate resolution cryo-electron microscopy (cryo-EM) reconstructions ${ }^{6-10}$. Although several late steps of translocation have been described, the level of detail achieved in these studies is insufficient to suggest a precise mechanism explaining fidelity of translocation. At the same time, understanding of the principles of the fidelity has become particularly crucial during the current viral pandemic, because programmed mRNA frameshifting is at the heart of the SARS-CoV-2 replication cycle ${ }^{11}$.

Here we present the crystal structure of eukaryotic $80 \mathrm{~S}$ ribosomes from Saccharomyces cerevisiae trapped in intermediate translocation state. The $X$-ray crystal structure provides a detailed mechanism of tRNA translocation from A- to P-sites and highlights the specific role of eEF2 in the movement of the tRNA-mRNA module during the process. The crystal structure presented here uncovers the precise role of diphthamide and wybutosine, a heavily modified nucleoside at position 37 of eukaryotic phenylalanine tRNA, in stabilization of the codon-anticodon interactions during translocation and demonstrates how eukaryote-specific elements of the $80 \mathrm{~S}$ ribosome, eEF2 and tRNA rearrange to ensure maintenance of the mRNA reading frame.

\section{Architecture of the translocation complex}

We determined the structure of the S. cerevisiae 80 S ribosome translocation complex trapped in intermediate state by X-ray crystallography at 3.2 Å resolution (Fig. 1a, b, Extended Data Fig. 1, Extended Data Table 1). It consists of $S$. cerevisiae $80 S$ ribosomes bound with native S. cerevisiae eEF2, the nonhydrolyzable GTP analogue GMPPCP, mRNA and two tRNAs, and was determined in the absence of antibiotics, which customarily used for stabilization, suggesting that the model represents a bona fide state of the $80 \mathrm{~S}$ ribosome.

The crystal structure of the ribosome complex represents an intermediate translocation state that has not been described before with two tRNAs trapped in the chimeric hybrid ap/P and pe/E transitory positions. In this state the small subunit (SSU) head has swivelled $13.5^{\circ}$ and the SSU body has undergone $9^{\circ}$ anticlockwise rotation relative to the large subunit (LSU) (Fig.1a). The anticodon stem-loop (ASL) of the A-site tRNA is captured half-translocated between the A- and P-sites of SSU $(12.4 \AA$ out of a fully translocated distance of $24.1 \AA$ ), and the tRNA acceptor end contacts the P-loop of the peptidyl-transferase centre of LSU forming an ap/P chimeric hybrid state (Fig. 1a, b, Extended Data Fig. 2a, b).

The overall conformation of eEF2 resembles that of eEF2 in the $80 \mathrm{~S}$ translocation intermediate-post-translocation (TI-POST)-1

${ }^{1}$ Institute of Genetics and Molecular and Cellular Biology, CNRS UMR7104, INSERM U1258, University of Strasbourg, Illkirch, Strasbourg, France. ${ }^{2}$ Urania Therapeutics, Ostwald, France.

${ }^{3}$ Present address: Biochemistry and Biophysics Center, National Heart, Lung and Blood Institute, Bethesda, MD, USA. ${ }^{凶}$--mail: marat@igbmc.fr; gula@igbmc.fr 

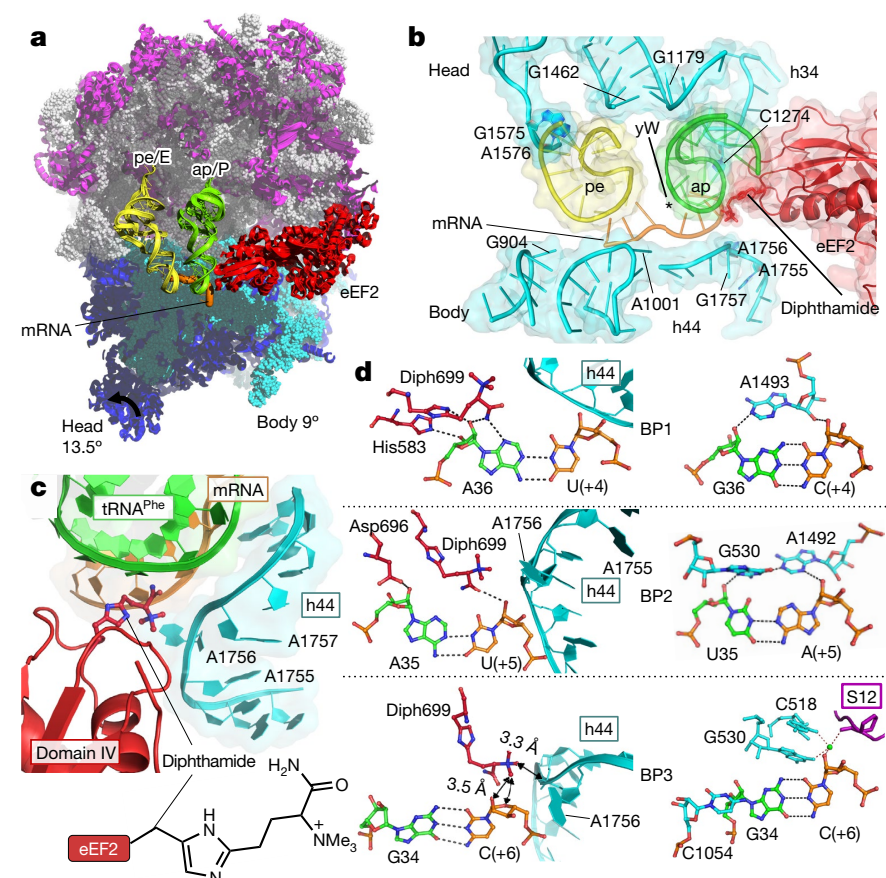

Fig.1|The translocation-intermediate state of the eukaryotic 80 S ribosome with EEF2-GMPPCP, mRNA and tRNAs,, showing diphthamide of eEF2 is involved in stabilizing codon-anticodon interactions early in translocation. a, Overview of the translocation-intermediate complex with two tRNAs trapped in chimeric hybrid ap/P and pe/Estates.b. Close-up view of ap/P and pe/EtRNA anticodon stem loops in the context of elements of the SSU body and head and domain IV of eEF2. Position of wybutosine ( $y W$ ) of ap/PtRNA ${ }^{\text {Phe }}$ is indicated by the asterisk.c, Position of diphthamide (Diph699) at the conjunction of the AAG anticodon of ap/PtRNA ${ }^{\text {Phe }}$, mRNA codon UUC and decoding adenosines 1755-1756 of helix 44 (h44).d, Stabilization networks around codon-anticodon interactions in the translocation-intermediate complex (left) and the decoding centre of the bacterial ribosome in the cognate classical state ${ }^{13,14}$ (right). Top, middle and bottom panels depict stabilization around the first, second and third base pairs (BP1-3) of a codon-anticodon duplex, respectively. Conserved adenosines 1755 and 1756 in yeast $18 \mathrm{~S}$ rRNA correspond to adenosines 1492 and 1493 in $16 \mathrm{~S} \mathrm{rRNA}$ of the bacterial decoding centre. eEF2 is shown in red, mRNA is in orange, chimeric hybrid ap/PtRNA is ingreen and chimeric hybrid pe/E is in yellow. LSU rRNA and proteins are shown in grey and purple; SSU rRNA and proteins are in cyan and in deep blue. Degrees of SSU body and head rotations are indicated and were obtained by superimposing with the non-rotated 80 S ribosome (PDB ID 3J78).

state of a medium-resolution cryo-EM reconstruction ${ }^{6}$ that depicts a late-translocation event with domain IV of eEF2 protruding approximately $7 \AA$ deeper into the A-site of SSU from the perspective of the SSU body (Extended Data Fig. 3). However-in contrast to the TI-POST-1 state-in our complex, domain IV of eEF2 is engaged in an extensive stabilization network with the codon-anticodon duplex and with the decoding centre area (Fig. 1b-d, Extended Data Fig. 1d). The intricate network of arginine-rich regions of domain II and III of eEF2 with helix 5 of 18S rRNA is formed owing to strong rotation of the SSU body $\left(9.85^{\circ}\right)$. These contacts are not present in the less rotated $\left(4.26^{\circ}\right)$ TI-POST-1 or unrotated TI-POST-3 states of 80 S from the cryo-EM models ${ }^{6}$ (Extended Data Fig. 4a,b). This comparison suggests that eEF2 uncouples from the SSU body at the later steps of translocation where it is associated only with LSU and remains stationary relative to the rest of the ribosome. Collectively, these structural data corroborate an existing hypothesis describing a translocase (eEF2 or EF-G) as a 'locking pawl' that decouples tRNA-mRNA from the SSU head and body during back-rotation ${ }^{3}$.

Comparison of the current early-intermediate state with late-translocation states described by cryo-EM ${ }^{6}$ reveals that the uS12 protein remains attached to domain III of eEF2 during SSU back-ratcheting. Located on the SSU shoulder, uS12 is implicated in translocation ${ }^{12}$ and codon-anticodon duplex stabilization in the decoding centre ${ }^{13}$. It is plausible that during translocation, uS12 transmits SSU body back-rotation by pulling domain III of eEF 2 that in turn retransmits it to switch II in the G-domain, stimulating GTP hydrolysis (Extended Data Fig. 4c, d). We found additional stabilization of domain IV by the N terminus of the eukaryote-specific protein eS30, which also interacts extensively with conserved decoding protein uS12 (Extended Data Fig. 4c). It can be assumed that eS30 co-evolved with eEF2, whose domain IV has 65 additional amino acids compared with its bacterial counterpart EF-G, to provide supplementary stabilization as well as enhancing propagation of conformational changes at the decoding site $^{13,14}$. Other interactions between eEF2, tRNAs and the 80 S ribosome are presented in Extended Data Figs. 5, 6.

\section{Diphthamide}

The current crystal structure of 80 S ribosome translocation intermediate reveals direct involvement of diphthamide in stabilization of a codonanticodon duplex in transition from the A- to P-site. eEF2 interacts with the mRNA codon exclusively via diphthamide, which protrudes into a cleft formed by mRNA, ap/P tRNA and rRNA (Fig. 1c, d). Assisted by His583 and Asp696 of eEF2, diphthamide contacts the codon-anticodon duplex minor groove. A similar pattern of interactions has been extensively described between the bacterial decoding centre mould and the codon-anticodon duplex in a classical unrotated state ${ }^{13,14}$ (Fig. 1d). The closest resemblance is observed at the second base pair (BP2, Fig.1d), wherediphthamidetogetherwithAsp696mimicsstabilizationof decoding nucleotides G577 and A1755 in 18S rRNA (bacterial G530 and A1492). Fixation of the first codon-anticodon pair is divided between diphthamide with His583 and His694 contacting the anticodon ribose, and the wybutosine modification of ap/P tRNA ${ }^{\text {Phe }}$ of nucleotide 37 stacking on codon position +4 (BP1, Figs. 1d, 2a). The third codon-anticodon pair (BP3, Fig. 1d) is anchored by diphthamide only through its trimethylammonio moiety stabilizing the codon position. These findings are corroborated by numerous studies describing increased -1 frameshifting slippage when translation is performed with eEF2 lacking diphthamide $\mathrm{e}^{1,15}$.

The mRNA backbone of the UUC codon paired to ap/P tRNA ${ }^{\text {Phe }}$ is located in close proximity to the sugar-phosphate backbone of decoding nucleotides A1755-A1756 and G1757, whose backbone is bulged out because of partial 'flipped-in' and 'flipped-out' positions of their nucleoside moieties (Fig. 2b). This close positioning of mRNA and A1756-G1757 backbones is realized by ribose-phosphate zipper interactions ${ }^{16}$. The diphthamide trimethylammonio moiety additionally stabilizes negatively charged backbones by interaction with the phosphate group between A1756 and G1757 of 18S rRNA (BP3, Fig. 1d).

The difference in structure of helix 44 of SSU rRNA (h44), which forms a part of the decoding centre between bacteria and both eukaryotes and archaeamightexplainemergenceof thediphthamidemodificationduring evolution (Extended Data Fig. 7). The changes of decoding centre bulge, and flanking nucleotides could lead to increased flexibility of the eukaryotic decoding centre, which would require additional stabilization that was achieved by development of diphthamide modification of eEF2. In addition, the unique trimethylammonio moiety could have been evolutionally refined to reduce repulsion between juxtaposing negatively charged backbones of mRNA and the h44 decoding-centre loop at the early stages of translocation.

\section{Unlocking of the decoding centre}

In the early-intermediate translocation state, when diphthamide takes over stabilization of the codon-anticodon duplex, decoding nucleotides rearrange to initiate resetting of the decoding centre. Nucleotide A1755 of 18S rRNA adopts a flipped-in position, whereas A1756 is in a more flipped-out and somewhat flexible conformation. There is a noticeable 


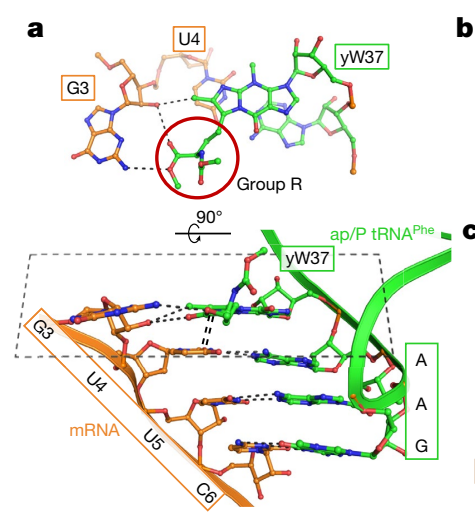

Fig. 2 |Stabilization of mRNA-tRNA by wybutosine modification of tRNA ${ }^{\text {Phe }}$, rearrangements of the decoding centre and depiction of a pawl function for eEF2 during translocation. a, Wybutosine cross-strand stacks on the first codon-anticodon base pair formed by mRNA codon UUC and $\mathrm{tRNA}^{\text {Phe }}$ at the ap/P state and stabilizes the last position of the adjacent upstream AUG codon (G3). An alternative view (bottom) of the same pattern clearly demonstrates how wybutosine enhances stabilization of mRNA to prevent a frame shift during translocation. $b$, Interactions between the backbones of translocating mRNA and decoding A1756 and A1757 of h44; mRNA in classical state is in grey. $c$, A shift of helix 69 (H69) of 25S rRNA is
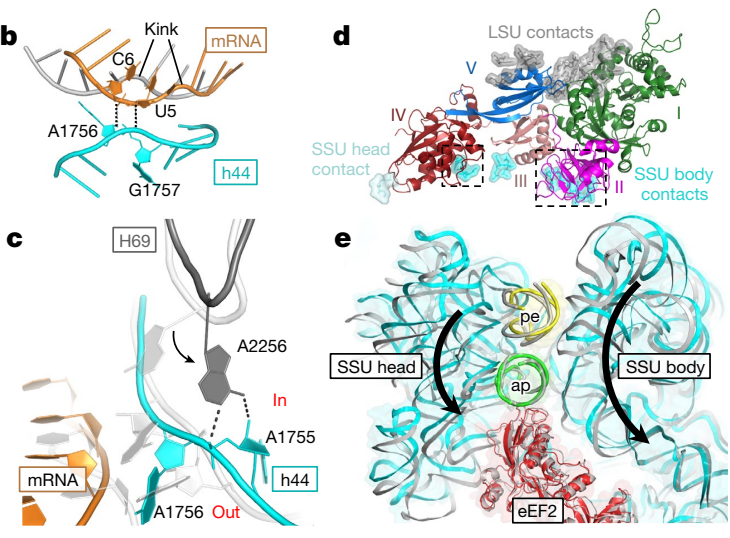

coupled to rearrangements of the decoding centre as compared to the bacterial classical state in white (PDB ID 4V6F). d, Contacts of eEF2 (coloured by domain) with LSU (grey), body (cyan) and head (pale cyan) of SSU; dashed squares indicate contacts that are disrupted at the late stage of translocation, allowing eEF2 to uncouple from the SSU body.e, Superposition of intermediateand late (grey) (TI-POST-1, PDB ID 6GZ3)-translocation structures relative to LSU reveals eEF2 acting as a pawl anchoring and decoupling ap/P tRNA from the SSU head and body. The reverse direction of the SSU head and body rotation to a classical post-translocation state is indicated with arrows. rearrangement of the pivotal intersubunit bridge $\mathrm{B} 2 \mathrm{a}$ that is partially composed of A2256 of LSU helix 69 (H69) and decoding nucleotides of h44 (A1755-A1756) of SSU (Fig. 2c). In the present structure, adenosine 2256 of H69 protrudes towards sugar phosphate backbone between A1755-A1756, and contacts A1756 phosphate and A1755 ribose moieties. This connection allows $\mathrm{H} 69$ to transmit its movement directly to decoding adenines.

During selection of tRNA on the ribosome, the decoding centre serves as a mould imposing restraints on codon-anticodon nucleotides via defined interactions ${ }^{13,14}$ (Fig.1d, right). To translocatemRNAby one codon, the decoding centre has to unlock from the bound codon-anticodon duplex. The present crystal structure with eEF2 suggests that destabilization of the decoding centre is initiated by anticlockwise rotation of SSU, which leads to rearrangement of the $\mathrm{B} 2 \mathrm{a}$ bridge. The latterrearrangement induces displacement of $\mathrm{H} 69$ towards decoding $\mathrm{h} 44$, resulting in a new contact of A2256 of $\mathrm{H} 69$ with the decoding nucleotides A1755-A1756. Further rearrangements of the $\mathrm{H} 69$ tip lead to its synchronized movement with the decoding adenosines and triggers partial unlocking of the decoding centre from mRNA and tRNA (Fig. 2c). The trimethylammonio moiety of diphthamide contributes to these changes of A1755-A1756 and prevents decoding adenines from re-establishing their contacts with the codon-anticodon duplex (Fig. 1c, d).

Diphthamide-induced unlocking catalyses translocation by reducing the energy required for movement of the codon-anticodon pair. This function is supported by biochemical studies, which have demonstrated decreased protein synthesis rates in organisms lacking diphthamide ${ }^{4,15}$. Such an interpretation is also consistent with results of pre-steady-state kinetics studies on the bacterial translocation system ${ }^{17}$ and may explain the inhibitory effects of the antibiotics paromomycin and viomycin that interfere with the resetting of the decoding nucleotides by binding to the decoding centre region of $\mathrm{h} 44$ and reduce the rate of translocation by about 160 - and more than 10,000 -fold, respectively ${ }^{18-20}$.

\section{Wybutosine}

In the current structure, we observe clear density for the tRNA ${ }^{\text {Phe }}$ hyper-modification wybutosine in position 37 in an authentic binding state on the ribosome. Wybutosine consists of wyosine base with 4-methoxy-3-[(methoxycarbonyl)amino]-4-oxobutyl group (group R; Fig. 2a, Extended Data Fig. 2c). Wyosine cross-strand stacks with the first codon nucleoside paired to ap/P tRNA. Group R stretches towards the third nucleotide (G3) of the codon coupled to the anticodon of pe/E tRNA and forms two hydrogen bonds between its methylcarboxyl portion and G3, hence, upgrading the triplet codon-anticodon interaction with ap/ P tRNA to a quadruplet interaction (Fig. 2a). To our knowledge, this is the first time a tRNA modification has been seen to directly influence two adjoint codon-anticodon pairs by interactions with mRNA. Interactions between group $\mathrm{R}$ of wybutosine and the third position of the adjacent codon coupled to pe/E tRNA are possible because of a closer distance between ap/P tRNA and pe/E tRNA (Extended DataFig. 2a). This situation is not achievable with tRNAs in classical $\mathrm{A} / \mathrm{A}$ and $\mathrm{P} / \mathrm{P}$ states and during the early stage of translocation where both tRNAs are positioned more than $10 \AA$ apart, indicating that the main stabilization role of wybutosine modification is strongly manifested in the intermediate translocation states. This also implies that the $(-1)$ ribosomal frameshifting events with hypomodified wybutosine tRNA are likely to occur during intermediate and late steps of translocation. These findings substantiate previous studies explaining why lack or alteration of wybutosine derivatives in tRNA ${ }^{\text {Phe }}$ increases the $(-1)$ programmed ribosomal frameshifting frequency and is associated with poor survival in patients with cancer ${ }^{2,21}$, and also demonstrates how the presence of wybutosine influences the efficiency of viral ribosomal frameshifting ${ }^{22}$.

\section{Conclusion}

The unique state of the translocating eukaryotic ribosome described here demonstrates eEF2 functioning as a pawl ${ }^{3}$ during translocation (Figs. 2d, 3, Extended Data Fig. 4a, b). Comparison of the early translocation-intermediate complex described here with the late translocation-intermediate TI-POST- $1^{6}$ shows that eEF 2 is sturdily anchored on LSU via domains I and V (Fig. 2e, Supplementary Video) with tRNAs retaining very similar positions in both states of the 80 S ribosome, while SSU head and body move around them. The comparison suggests that domain IV of eEF2 uncouples tRNA-mRNA from the SSU body and head allowing these domains to return to their pretranslocation positions without pulling tRNA and mRNA back with them. Interestingly, an increase in the head swivel from around $13.5^{\circ}$ (in the early intermediate) to about $18^{\circ}$ (in TI-POST-1) is a result of the body back-rotation that the head cannot follow because it remains attached to tRNAs (Fig. 2e). 


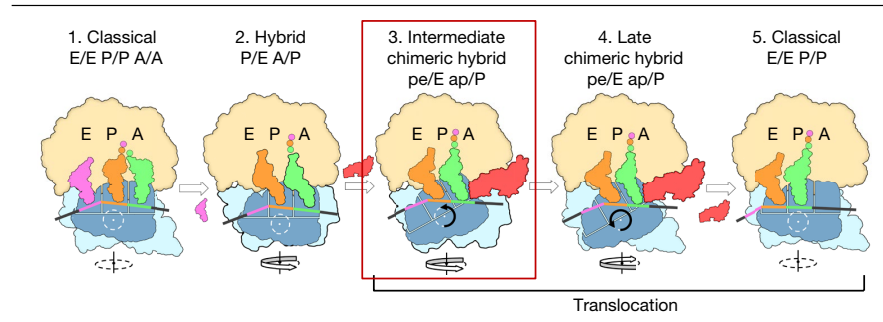

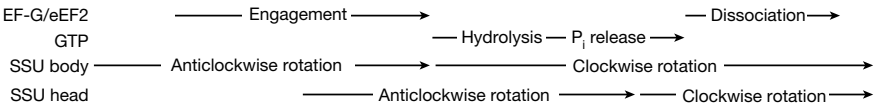

Fig. 3 | Integrating kinetic and structural studies of translocation.

Top, translocation scheme based on the crystal structure of the intermediate translocation complex reported here (in frame) and on cryo-EM structures of late translocation (PDB ID: 6GZ3 and 6GZ5), as well as hybrid and classical post-translocation states (PDB ID: 3J77 and 3J78). A proposed sequence of events based on kinetic studies ${ }^{28}$ is shown at the bottom. Steps 1 and 2 , thermally driven intersubunit rotations lead to tRNAs adopting hybrid $\mathrm{A} / \mathrm{P}$ and P/E states and EEF2-GTP binding to the 80 S ribosome. Steps 2 and 3 , concomitant changes of LSU H69 composing intersubunit bridge B2a and the decoding centre, and insertion of the eEF2 diphthamide to the SSU A-site induce unlocking of the decoding centre. The released codon-anticodon duplex becomes stabilized by direct interactions with diphthamide. Detachment of tRNA ASLs from the SSU body and further insertion of the eEF2 domain IV into the A-site cause initial anticlockwise rotation of the head and movement of the second tRNA from the SSU P-site towards the E-site where it binds to L1 stalk. Steps 3 and 4, eEF2 remains anchored to LSU via domains I and $V$ but is released from SSU where domain IV uncouples tRNA-mRNA from rearrangements of the SSU body and head. What is perceived as an additional large swivelling of the head is actually a result of the body back-rotation while the head remains fixed to $t R N A s$. Step 4 and 5 , this body rotation increases the strain in the SSU neck and leads to uncoupling of the head from tRNAs. Formation of contacts between rRNA of the head and domain IV of eEF2 restrain the head position. The last steps of translocation are achieved when the head, owing to the increasing strain on the neck, snaps back to a non-rotated state and tRNA-mRNA binds to the SSU P-site environment.

This probably creates a strain in the SSU neck region that eventually forces the head to unbind from tRNAs and to rotate back (Supplementary Video). Thus, the last steps of translocation are achieved by back retracement of the head to a non-rotated state and rebinding of tRNA-mRNA (one codon further down) to the SSU P-site environment.

The translocation process is enabled by Brownian intersubunit rotations of the ribosome $\mathrm{e}^{23,24}$. The GTP-bound state of eEF2 on the ribosome stays in rigid conformation, as it is observed in current structure of early-translocation intermediate as well as in late-translocation intermediates $^{6}$ (Fig. 2d, e), thus serving as a pawl that ensures directionality of translocation process. The described model supports findings showing that the energy stored in the eEF2•GTP state is sufficient to promote translocation $^{25}$ and suggests that hydrolysis of GTP does not occur until the late steps of the process. It has been shown previously that domain IV of eEF2-GTP state can reach a codon-anticodon duplex of the P-site tRNA of the non-rotated ribosome ${ }^{6}$. However, experiments using fluorescence resonance energy transfer and other techniques ${ }^{6,26}$ have reported that translocation induced by eEF 2 with a non-hydrolyzable analogue of GTP is prone to reversion, demonstrating a critical role of GTP hydrolysis in promoting unidirectionality of translocation. GTP hydrolysis and inorganic phosphate release are most likely to occur during the late steps of translocation during SSU body back-ratcheting, when hydrolysis of GTP is stimulated by the movement of uS12-eEF2 domain III (Extended Data Fig. 4c, d). Conformational changes of eEF2 induced by GTP hydrolysis enable unbinding from the mRNA-tRNA module in a manner that prevents pulling the codon-anticodon duplex back to the A-site. Similarly, the bacterial homologue of eEF2 undergoes a large rotation in domain III before its dissociation from the ribosome ${ }^{27}$.

\section{Online content}

Any methods, additional references, Nature Research reporting summaries, source data, extended data, supplementary information, acknowledgements, peer review information; details of author contributions and competing interests; and statements of data and code availability are available at https://doi.org/10.1038/s41586-021-04131-9.

1. Ortiz, P. A., Ulloque, R., Kihara, G. K., Zheng, H. \& Kinzy, T. G. Translation elongation factor 2 anticodon mimicry domain mutants affect fidelity and diphtheria toxin resistance. J. Biol. Chem. 281, 32639-32648 (2006).

2. Waas, W. F., Druzina, Z., Hanan, M. \& Schimmel, P. Role of a tRNA base modification and its precursors in frameshifting in eukaryotes. J. Biol. Chem. 282, 26026-26034 (2007).

3. Noller, H. F., Lancaster, L., Mohan, S. \& Zhou, J. Ribosome structural dynamics in translocation: yet another functional role for ribosomal RNA. Q. Rev. Biophys. 50, e12 (2017)

4. Murray, J. et al. Structural characterization of ribosome recruitment and translocation by type IV IRES. Elife 5, e13567 (2016).

5. Jørgensen, R., Merrill, A. R. \& Andersen, G. R. The life and death of translation elongation factor 2. Biochem. Soc. Trans. 34, 1-6 (2006).

6. Flis, J. et al. tRNA translocation by the eukaryotic 80 s ribosome and the impact of GTP hydrolysis. Cell Rep. 25, 2676-2688.e7 (2018).

7. Pellegrino, S. et al. Structural insights into the role of diphthamide on elongation factor 2 in mRNA reading-frame maintenance. J. Mol. Biol. 430, 2677-2687 (2018).

8. Spahn, C. M. et al. Domain movements of elongation factor eEF2 and the eukaryotic $80 \mathrm{~S}$ ribosome facilitate tRNA translocation. EMBO J. 23, 1008-1019 (2004).

9. Taylor, D. J. et al. Structures of modified eEF2 $80 \mathrm{~S}$ ribosome complexes reveal the role of GTP hydrolysis in translocation. EMBO J. 26, 2421-2431 (2007).

10. Voorhees, R. M., Fernández, I. S., Scheres, S. H. W. \& Hegde, R. S. Structure of the mammalian ribosome-Sec61 complex to 3.4 Å resolution. Cell 157, 1632-1643 (2014).

11. Bhatt, P. R. et al. Structural basis of ribosomal frameshifting during translation of the SARS-CoV-2 RNA genome. Science 372, 1306-1313 (2021).

12. Gavrilova, L. P., Koteliansky, V. E. \& Spirin, A. S. Ribosomal protein S12 and 'non-enzymatic' translocation. FEBS Lett. 45, 324-328 (1974).

13. Ogle, J. M. et al. Recognition of cognate transfer RNA by the 30 S ribosomal subunit. Science 292, 897-902 (2001).

14. Demeshkina, N., Jenner, L., Westhof, E., Yusupov, M. \& Yusupova, G. A new understanding of the decoding principle on the ribosome. Nature 484, 256-259 (2012).

15. Liu, S. et al. Diphthamide modification on eukaryotic elongation factor 2 is needed to assure fidelity of mRNA translation and mouse development. Proc. Natl Acad. Sci. USA 109, 13817-13822 (2012).

16. Ulyanov, N. B. \& James, T. L. RNA structural motifs that entail hydrogen bonds involving sugar-phosphate backbone atoms of RNA. New J. Chem. 34, 910-917 (2010).

17. Khade, P. K. \& Joseph, S. Messenger RNA interactions in the decoding center control the rate of translocation. Nat. Struct. Mol. Biol. 18, 1300-1302 (2011).

18. Prokhorova, I. et al. Aminoglycoside interactions and impacts on the eukaryotic ribosome. Proc. Natl Acad. Sci. USA 114, E10899-E10908 (2017).

19. Stanley, R. E., Blaha, G., Grodzicki, R. L., Strickler, M. D. \& Steitz, T. A. The structures of the anti-tuberculosis antibiotics viomycin and capreomycin bound to the $70 S$ ribosome. Nat. Struct. Mol. Biol. 17, 289-293 (2010).

20. Peske, F., Savelsbergh, A., Katunin, V. I., Rodnina, M. V. \& Wintermeyer, W. Conformational changes of the small ribosomal subunit during elongation factor G-dependent tRNA-mRNA translocation. J. Mol. Biol. 343, 1183-1194 (2004).

21. Rosselló-Tortella, M. et al. Epigenetic loss of the transfer RNA-modifying enzyme TYW2 induces ribosome frameshifts in colon cancer. Proc. Natl Acad. Sci. USA 117, 2078520793 (2020).

22. Carlson, B. A. et al. Transfer RNA modification status influences retroviral ribosomal frameshifting. Virology 255, 2-8 (1999).

23. Moazed, D. \& Noller, H. F. Intermediate states in the movement of transfer RNA in the ribosome. Nature 342, 142-148 (1989).

24. Cornish, P. V., Ermolenko, D. N., Noller, H. F. \& Ha, T. Spontaneous intersubunit rotation in single ribosomes. Mol. Cell 30, 578-588 (2008).

25. Salsi, E., Farah, E. \& Ermolenko, D. N. EF-G activation by phosphate analogs. J. Mol. Biol. 428, 2248-2258 (2016).

26. Susorov, D. et al. Eukaryotic translation elongation factor 2 (eEF2) catalyzes reverse translocation of the eukaryotic ribosome. J. Biol. Chem. 293, 5220-5229 (2018).

27. Chen, $\mathrm{C}$. et al. Elongation factor $\mathrm{G}$ initiates translocation through a power stroke. Proc. Natl Acad. Sci. USA 113, 7515-7520 (2016).

28. Belardinelli, R. et al. Choreography of molecular movements during ribosome progression along mRNA. Nat. Struct. Mol. Biol. 23, 342-348 (2016).

Publisher's note Springer Nature remains neutral with regard to jurisdictional claims in published maps and institutional affiliations.

Open Access This article is licensed under a Creative Commons Attribution 4.0 International License, which permits use, sharing, adaptation, distribution and reproduction in any medium or format, as long as you give appropriate credit to the original author(s) and the source, provide a link to the Creative Commons license, and indicate if changes were made. The images or other third party material in this article are included in the article's Creative Commons license, unless indicated otherwise in a credit line to the material. If material is not included in the article's Creative Commons license and your intended use is not permitted by statutory regulation or exceeds the permitted use, you will need to obtain permission directly from the copyright holder. To view a copy of this license, visit http://creativecommons.org/licenses/by/4.0/.

(c) The Author(s) 2021 


\section{Methods}

\section{$80 S$ ribosome purification}

Purification of the $80 S$ ribosomes from the JD1370- $\Delta$ Stm1 yeast strain $^{7}$ was carried out according to the previously described protocol ${ }^{29}$, with some modifications. The crude ribosomes obtained by precipitation with $8.5 \% \mathrm{PEG} 20,000$ were re-suspended in buffer $\mathrm{M}$ (30 mM Hepes- $\mathrm{KOH}, 10 \mathrm{mM} \mathrm{MgCl}_{2}, 50 \mathrm{mM} \mathrm{KCl}, 8.5 \%$ mannitol, $0.5 \mathrm{mM}$ EDTA-KOH, $2 \mathrm{mM} \mathrm{DTT}, \mathrm{pH} 7.55)$ and $\mathrm{MgCl}_{2}$ and $\mathrm{KCl}$ concentrations were slowly adjusted to 10 and $500 \mathrm{mM}(10 / 500)$, respectively. The ribosomal suspension was then incubated on ice for $35 \mathrm{~min}$ with mild vortexing. The ribosomes were applied on the $6 \%$ sucrose cushion, which was prepared in the same dissociation conditions (10/500) and layered over the $10-30 \%$ sucrose gradient as in ref. $^{7}$ with $5 \mathrm{mM}$ spermidine $(5 \mathrm{mg}$ of ribosomes per SW28 tube). After the overnight centrifugation selected fractions of the $80 \mathrm{~S}$ ribosomes were collected and the ribosomes were precipitated by PEG 20,000.

\section{Purification of native eEF2}

The isolation procedure of native eEF2 was mainly based on the previously described protocol ${ }^{30}$, with changes in several steps. First, we used a fresh culture of yeast strain JD1370- $\Delta$ Stm1 grown to an $A_{600}$ of 5-6 and cells were lysed in a microfluidizer. Second, instead of S-Sepharose, source-Q and uno-Q ion-exchange columns we used SP-Sepharose, Q-Sepharose and introduced a gel filtration with Sephadex-200 as the final purification step. The final sample was stored in $\mathrm{pH} 7.5 \mathrm{buffer}$ consisting of $20 \mathrm{mM}$ Tris- $\mathrm{HCl}, 5 \mathrm{mM} \mathrm{MgCl}_{2}, 100 \mathrm{mM} \mathrm{KCl}, 10 \%$ glycerol and $1 \mathrm{mMDTT}$.

\section{Purification and aminoacylation of tRNAs}

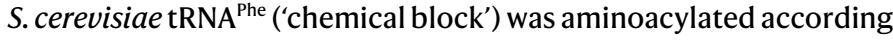
to the protocol as described ${ }^{31}$ with minor modifications. After three rounds of phenol-chloroform-isoamyl alcohol extraction S. cerevisiae Phe-tRNA ${ }^{\text {Phe }}$ was purified on a column DeltaPack, C4 300A, $5 \mathrm{mkm}$, $3.9 \times 150 \mathrm{~mm}$ HPLC column (Waters) using a ethanol gradient as described ${ }^{32}$. The final sample was stored in $20 \mathrm{mM} \mathrm{NH}_{4} \mathrm{CH}_{3} \mathrm{CO}_{2} \mathrm{pH}$ 5.0 at $-80^{\circ} \mathrm{C}$. Escherichia coli tRNA ${ }^{\mathrm{fmet}}$ was prepared and then aminoacylated and formylated according to ${ }^{33}$. After phenol extraction, fMet-tRNA ${ }^{\text {fmet }}$ was purified by hydrophobic chromatography using TSK-gel Phenyl-5PW column, and the final sample was stored in $20 \mathrm{mM}$ $\mathrm{NH}_{4} \mathrm{CH}_{3} \mathrm{CO}_{2} \mathrm{pH} 5.0$ at $-80^{\circ} \mathrm{C}$.

\section{S ribosome translocation complex formation}

For reconstitution of translocation complex S. cerevisiae 80 S ribosomes $(2.2 \mu \mathrm{M})$ and $5^{\prime}$-AAUGUUCAA-3' mRNA (Dharmacon) $(2.9 \mu \mathrm{M})$ were incubated at $30^{\circ} \mathrm{C}$ for $10 \mathrm{~min}$ in $6 \mathrm{mM} \mathrm{Mg}\left(\mathrm{CH}_{3} \mathrm{COO}\right)_{2}, 50 \mathrm{mM}$ $\mathrm{KOAc}, 10 \mathrm{mM} \mathrm{NH}_{4} \mathrm{Cl}$ and $1.25 \mathrm{mM} \mathrm{DTT}, 10 \mathrm{mM}$ Hepes- $\mathrm{KOH}$ (pH 7.5). The fMet-tRNA ${ }^{\text {fMet }}(2.9 \mu \mathrm{M})$ was added and the complex further incubated for $7 \mathrm{~min}$ at $30^{\circ} \mathrm{C}$ with following addition of Phe-tRNA $\mathrm{A}^{\text {Phe }}$ $(6.5 \mu \mathrm{M})$. The complex was incubated for additional $7 \mathrm{~min}$ at $30^{\circ} \mathrm{C}$. Separately, S. cerevisiae eEF2 $(8.7 \mu \mathrm{M})$ was incubated with GDPCP (Jena Bioscience) $(0.25 \mathrm{mM})$ for $10 \mathrm{~min}$ at room temperature and mixed with the ribosome complex for a final incubation at $30^{\circ} \mathrm{C}$ for $10 \mathrm{~min}$. The detergent Deoxy Big CHAP (CalBioChem) $(2.4 \mathrm{mM}$ ) was added and after $5 \mathrm{~min}$ at room temperature the complex was incubated at $4{ }^{\circ} \mathrm{C}$ for $5 \mathrm{~min}$.

\section{Crystallization and crystal treatment}

The $80 \mathrm{~S}$ ribosome translocation complex was crystallized at $4{ }^{\circ} \mathrm{C}$ by vapour diffusion in the MRC-48 siting drop plates (Hampton Research) by mixing $3 \mu$ lof the complex with $3 \mu$ of the reservoir solution $\left(100 \mathrm{mM}\right.$ bis-Tris-HCl, pH 7.0, $300 \mathrm{mM} \mathrm{NH}_{4} \mathrm{SCN}, 100 \mathrm{mM} \mathrm{KCl}$, $8.25 \%-9.5 \% \mathrm{PEG} 2 \mathrm{OK}, 1 \mathrm{mM} \mathrm{Mg}\left(\mathrm{CH}_{3} \mathrm{COO}\right)_{2}, 2 \%$ glycerol, $1 \%$ sucrose, $5 \mathrm{mM}$ putrescine). Crystals appeared after 3 days and grew to their full size in 13 days.
The post-crystallization treatment was carried out via dehydration by replacing reservoir solution with saturated $\mathrm{MgCl}_{2}$ salt and incubating for $18 \mathrm{~h}$. Treatment solution (3.3\% PEG 20K, 6\% PEG 10K, $115 \mathrm{mM}$ bis-Tris- $\mathrm{HCl}$, $\mathrm{pH} 5.4,18 \mathrm{mM}$ putrescine, $21 \mathrm{mM} \mathrm{Mg}\left(\mathrm{CH}_{3} \mathrm{COO}\right)_{2}$, 9\% glycerol, $0.75 \%$ sucrose, $1.8 \mathrm{mM}$ deoxy big CHAP, $2.3 \mathrm{mM} \mathrm{DTT}$ ) was added to the crystallization drop before dehydration. The crystals were collected and flash-frozen in liquid nitrogen.

\section{Data collection and structure refinement}

Data collection was carried out at $100 \mathrm{~K}$ at beamline PX1 - X06SA at the Swiss Light Source synchrotron at $1.0 \AA$ wavelength using DA+ data acquisition and analysis software ${ }^{34}$. Data were integrated and scaled with the XDS program ${ }^{35}$. The search model was generated from the previously published structure of the yeast 80 s ribosome ${ }^{29}$ (PDB ID 4V88). The initial molecular replacement solution was refined in PHENIX by rigid-body refinement with the $40 \mathrm{~S}$ and $60 \mathrm{~S}$ subunits treated as rigid bodies. After initial refinement, density corresponding to the mRNA, tRNAs, eEF2 as well as ribosome rearrangements became clearly visible in the difference electron density map. The crystal structure of eEF2 (PDB ID 1NOU) was docked into the density and manually adjusted before refinement. Refinement was carried out in alternating cycles of automated refinements using PHENIX with manual refinement and model building in COOT resulting in a model with Ramachandran favoured, allowed and outliers of $83.4 \%, 14.4 \%$ and $1.1 \%$ respectively. A summary of refinement and data collection statistics is displayed in Extended Data Table 1. All figures were generated using PyMOL.

\section{Reporting summary}

Further information on research design is available in the Nature Research Reporting Summary linked to this paper.

\section{Data availability}

Coordinates and structure factors have been deposited with the Protein Data Bank under accession code 7OSM. Previously published models that were used for analysis and comparison are also available in the Protein Data Bank with accession codes $4 \mathrm{~V} 88,4 \mathrm{~V} 6 \mathrm{~F}, 1 \mathrm{NOU}, 3 \mathrm{~J} 77,3 \mathrm{~J} 78$, $4 \mathrm{~V} 6 \mathrm{~F}, 6 \mathrm{GZ3}$ and $6 \mathrm{GZ5}$.

29. Ben-Shem, A., Jenner, L., Yusupova, G. \& Yusupov, M. Crystal structure of the eukaryotic ribosome. Science 330, 1203-1209 (2010).

30. Jørgensen, R., Carr-Schmid, A., Ortiz, P. A., Kinzy, T. G. \& Andersen, G. R. Purification and crystallization of the yeast elongation factor eEF2. Acta Crystallogr. D Biol. Crystallogr. 58, 712-715 (2002).

31. Spirin, A. S., Belitsina N. V. \& Yusupova, G. Z. Ribosomal synthesis of polypeptides from aminoacyl-tRNA without polynucleotide template. Methods Enzymol. 164, 631-649 (1988).

32. Mesters, J. R., Vorstenbosch, E. L. H., de Boer, A. J. \& Kraal, B. Complete purification of tRNA, charged or modified with hydrophobic groups, by reversed-phase high-performance liquid chromatography on a C4/C18 column system. J. Chromatogr. A 679, 93-98 (1994).

33. Mechulam, Y., Guillon, L., Yatime, L., Blanquet, S. \& Schmitt, E. Protection-based assays to measure aminoacyl-tRNA binding to translation initiation factors. Methods Enzymol. 430, 265-281 (2007).

34. Wojdyla, J. A et al. DA+ data acquisition and analysis software at the Swiss Light Source macromolecular crystallography beamlines. J. Synchrotron Radiat. 25, 293-303 (2018).

35. Kabsch, W. Automatic processing of rotation diffraction data from crystals of initially unknown symmetry and cell constants. J. Appl. Crystallogr. 26, 795-800 (1993).

36. Afonine, P. V. et al. FEM: feature-enhanced map. Acta Crystallogr. D Biol. Crystallogr. 71, 646-666 (2015)

37. Zhou, J., Lancaster, L., Donohue, J. P. \& Noller, H. F. Spontaneous ribosomal translocation of mRNA and tRNAs into a chimeric hybrid state. Proc. Natl Acad. Sci. USA 116, 7813-7818 (2019).

Acknowledgements We thank the staff of the PXI beamline at the Swiss Light Source (Switzerland) and M. Wang for assistance during synchrotron data collection; N. Milicevic for the preparation of aminoacylated tRNAs samples; and I. Prokhorova for assistance with purification of the $80 \mathrm{~S}$ ribosome and eEF2. Grant ANR-10-LABX-0030-INRT from a French State Fund managed by the ANR under the frame program Investissements d'Avenir ANR- 


\section{Article}

10-IDEX-0002-02 (to M.D.). M.D. started working on the project as an IGBMC International PhD

Programme fellow supported by LabEx INRT funds. This work was supported by the French

National Research Agency ANR-15-CE11-0021-01 (to G.Y.) and La Fondation pour la Recherche Médicale DEQ20181039600 (to M.Y.).

Author contributions M.D. and N.D. conducted biochemical and crystallization experiments. M.D., N.D., L.J. and A.R. participated in data collection. M.D., L.J. and A.R. performed molecula model building and refinement. Main data analysis was performed by M.D. N.D., L.J. and A.R. also contributed to the data analysis. M.Y. and G.Y. conceived and supervised the project. All authors discussed the results and commented on the manuscript.
Competing interests The authors declare no competing interests

Additional information

Supplementary information The online version contains supplementary material available at https://doi.org/10.1038/s41586-021-04131-9.

Correspondence and requests for materials should be addressed to Marat Yusupov or

Gulnara Yusupova.

Peer review information Nature thanks the anonymous reviewer(s) for their contribution to the peer review of this work.

Reprints and permissions information is available at http://www.nature.com/reprints. 

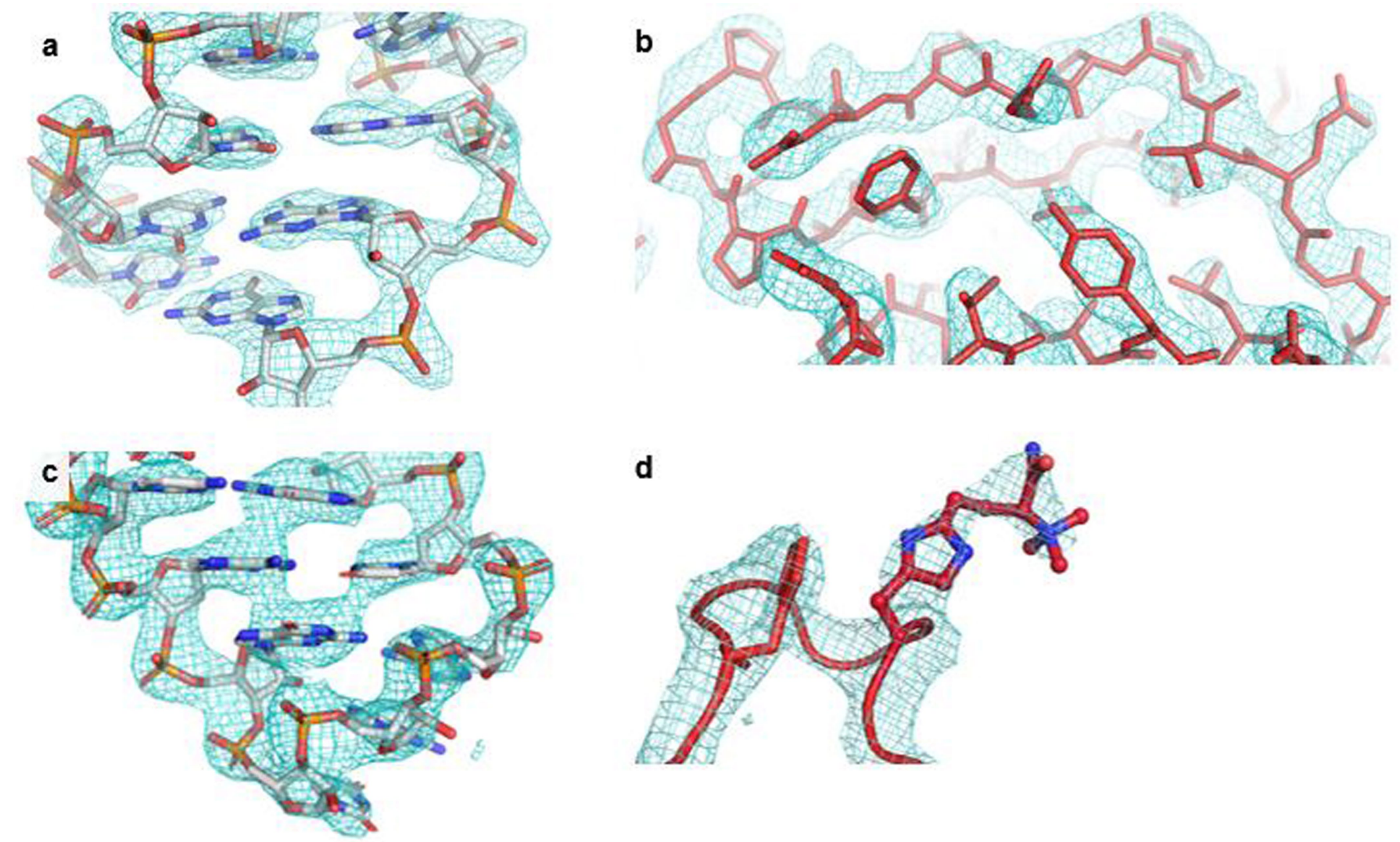

Extended Data Fig. 1 | Examples of the electron density maps of the $80 \mathrm{~S}$ ribosome translocation intermediate complex. $\mathrm{FEM}^{36}$ map for a

$2 F_{o}-F_{c}$ electron density map contoured at 1.5 sigma for $25 \mathrm{~S}$ rRNA (c) and at 1 sigma for the diphthamide loop of eEF2 (d). 
Article

a

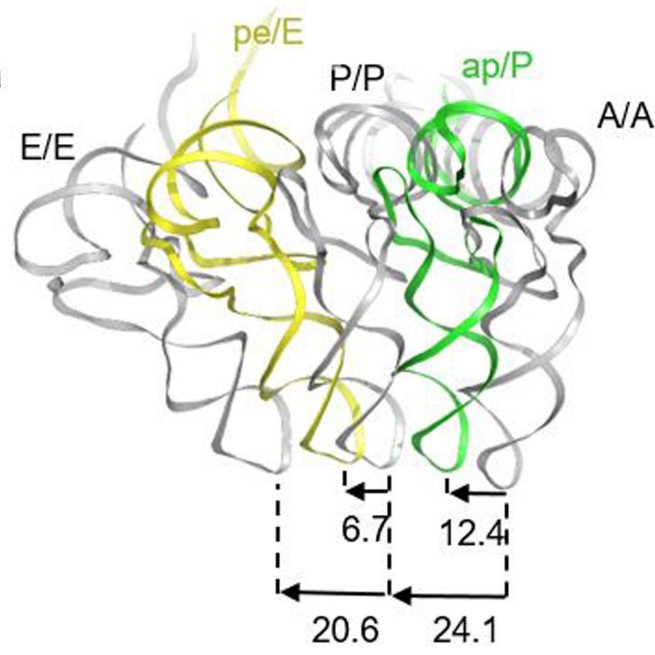

Extended Data Fig. 2 | Comparison of various states of tREAs on the ribosome. a, Positions of tRNAs of the intermediate translocation complex relative to tRNAs in the classical A, P and E states (grey, PDB ID 4V6F) aligned on
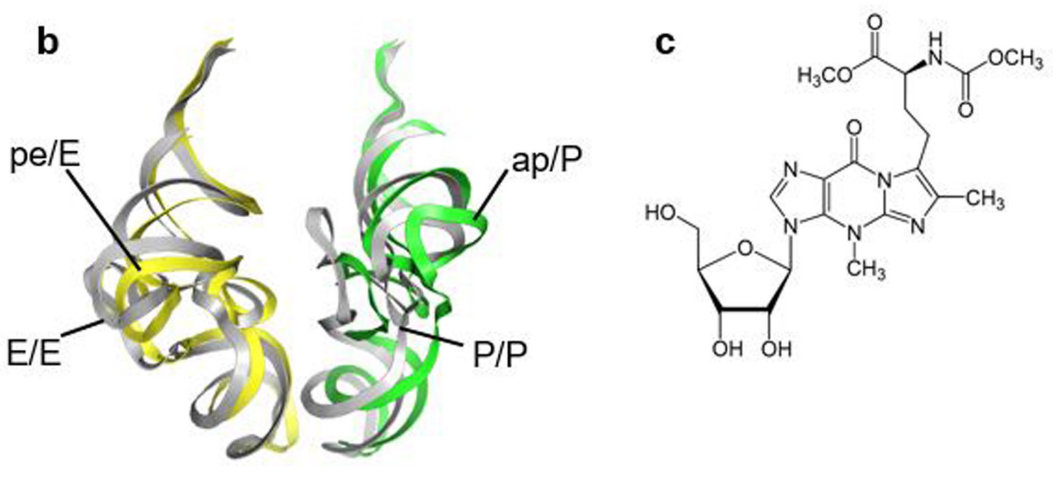

the SSU body; indicated distances are in A. b, Positions of tRNAs relative to tRNAs in the classical nonrotated state (PDB ID 3J78) aligned on LSU. c, Chemical structure of the wybutosine ERNA ${ }^{\text {Ph }}$ modification at position 37 . 


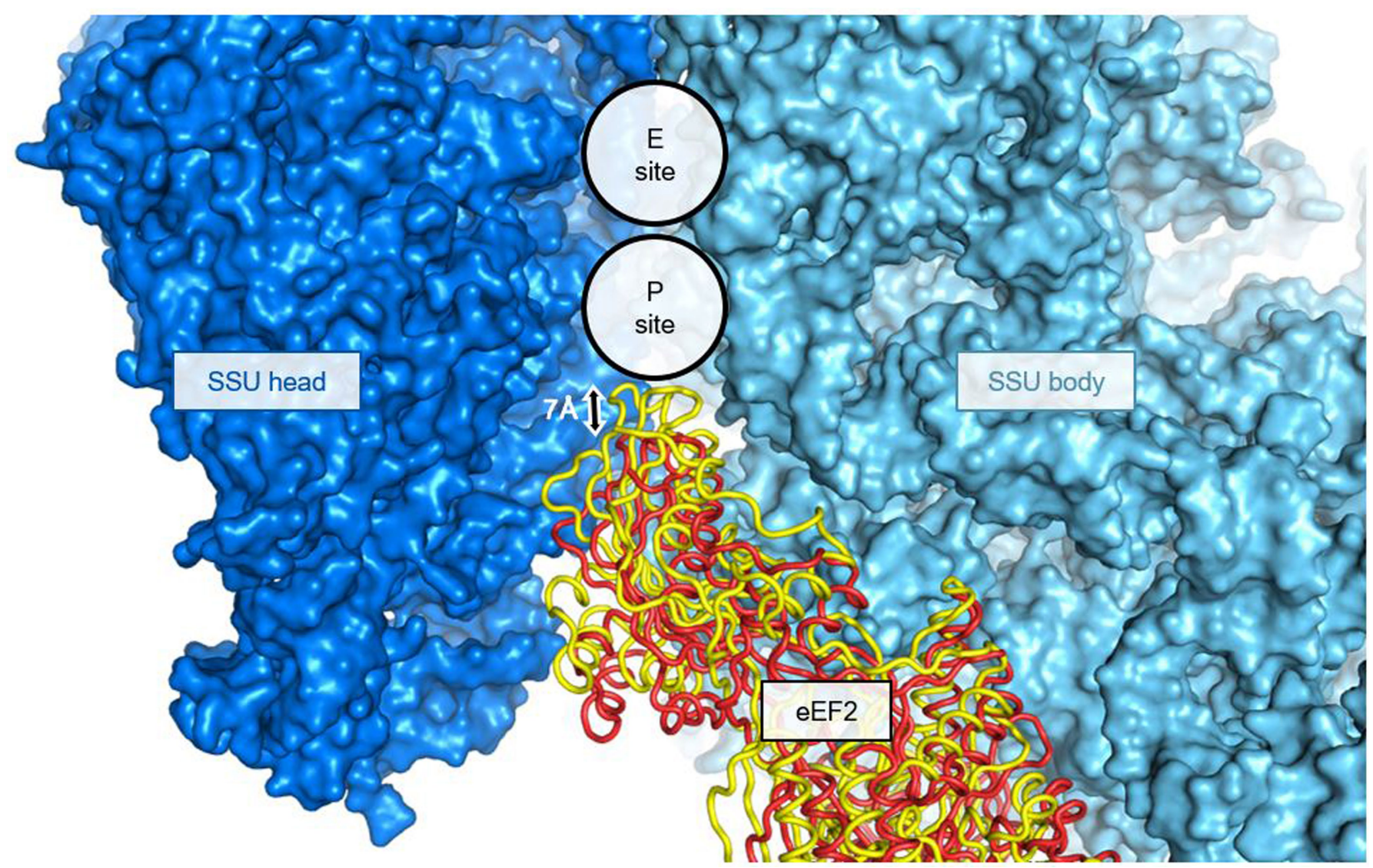

Extended Data Fig. 3 | Insertion of eEF2 into the SSU A site in the late translocation complex TI-POST1 (yellow, PDB ID 6GZ3) in comparison to the position of eEF2 in the early translocation intermediate state that we determined in this study (red). View from the LSU side. Alignment is performed using the SSU body. 
a

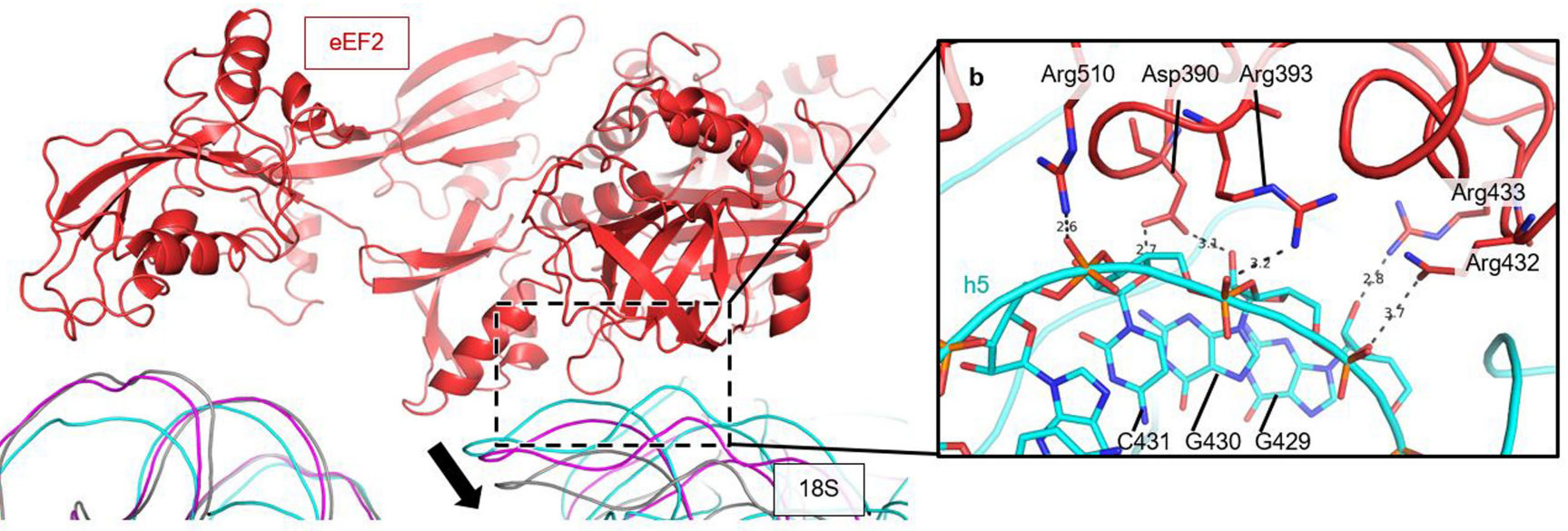

c

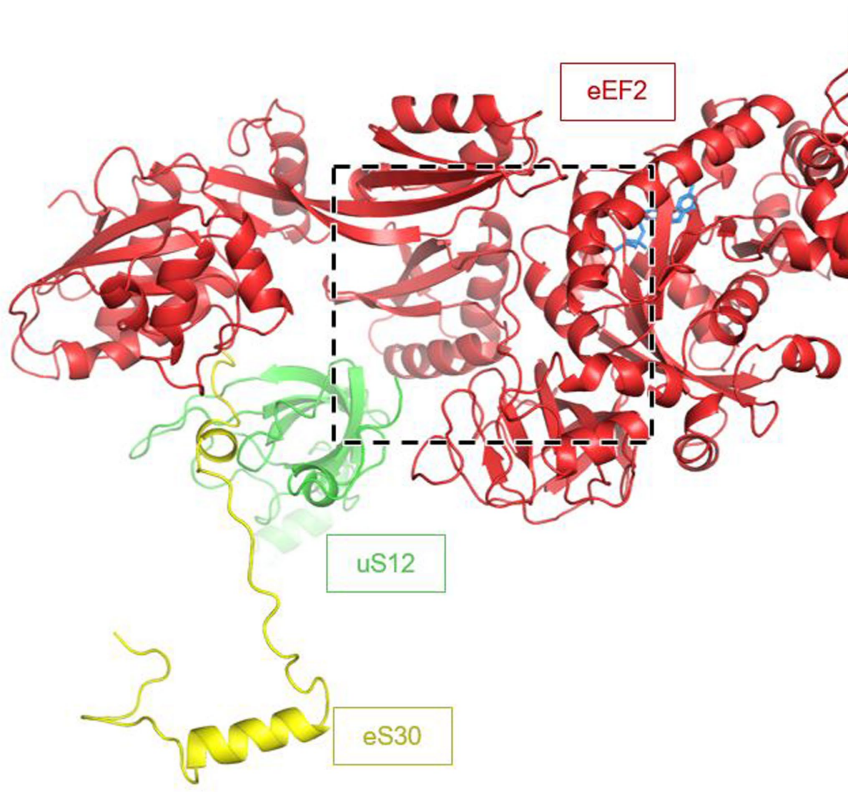

Extended Data Fig. 4 | Interactions of eEF2 with the SSU body. a, Comparison of the current X-ray structure of an early translocation intermediate (18S rRNA in cyan) with cryo-EM models of late translocation steps TI-POST1 (magenta, PDB ID 6GZ3) and TI-POST3 (grey, PDB ID 6GZ3) ${ }^{6}$. Superimposition of eEF2 in the two structures shows that there is a noticeable shift of $18 \mathrm{~S}$ rRNA of the SSU body away from eEF2 at later stages of translocation.b. Contacts between eEF 2 and h5 of SSU of the rotated ribosome in the reported intermediate translocation complex. c, Interactions between eEF 2 and the SSU shoulder proteins eS 30 and uS12. Stabilization of the eEF2 domain IV by the N-terminus of eukaryote-specific protein eS30 that itself interacts with conserved decoding protein uS12. Presumably, eS30 co-evolved with eEF2, whose domain IV has 65 additional

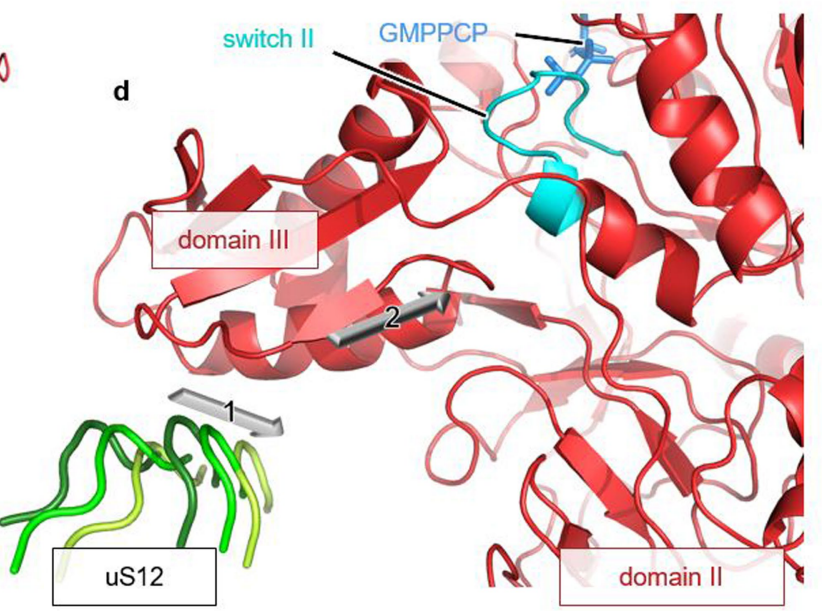

amino acids compared to its bacterial counterpart EF- $\mathrm{G}^{5}$, to provide supplementary stabilization as well as to enhance propagation of conformational changes at the decoding $\operatorname{sit}^{13,14}$.d, A close up view of the dashed region from (c). Movement of uS12 (arrow1) induced by the SSU body back-rotation can propagate to switch II (in cyan) of eEF2 through domain III (arrow 2) and can trigger GTP hydrolysis or Pi release. Regions of uS12 adjacent to eEF2 are shown and coloured dark- to light-green based on the rotational state where dark-green is the most rotated state (intermediate translocation complex) and medium (TI-POST1) and light (TI-POST3) greens represent the least rotated states. Alignment was done using eEF2. 
a

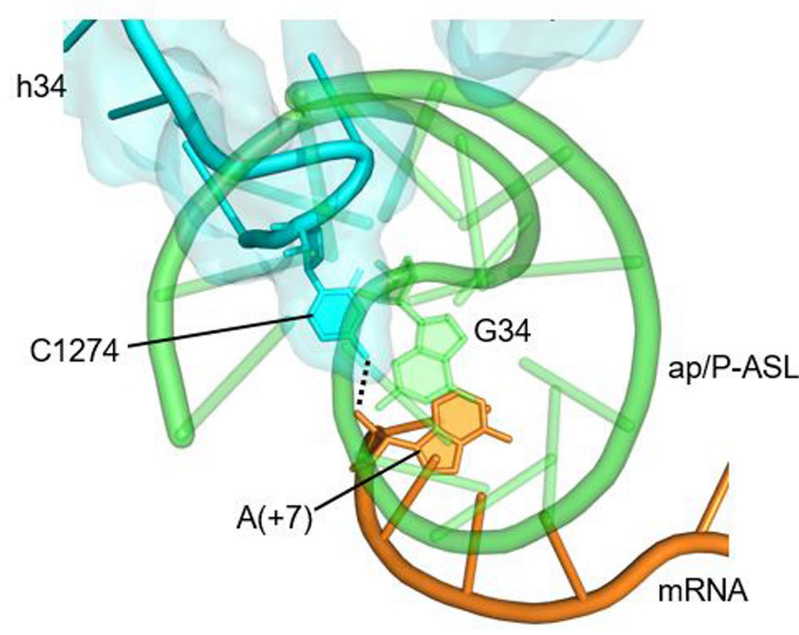

Extended Data Fig. 5 | Rearrangement of $h 34$ and $h 31$ of SSU with ASL movement of tRNAs in the translocation intermediate complex. $a$, A new position of conserved C1274 in h34 of 18S rRNA (C1054 of 16S rRNA in bacteria, Fig. 1d) relative to ASL of the A site tRNA and mRNA. By establishing a contact with mRNA nucleotide in position ( +7$) \mathrm{C} 1274$ can contribute to maintaining mRNA reading frame during translocation. $\mathbf{b}$, In the early intermediate state of translocation $\mathrm{h} 31$ of $18 \mathrm{~S}$ rRNA moves together with pe/E tRNA. In this state, the U1191-C1637 interaction is broken, however, U1191 remains in contact with

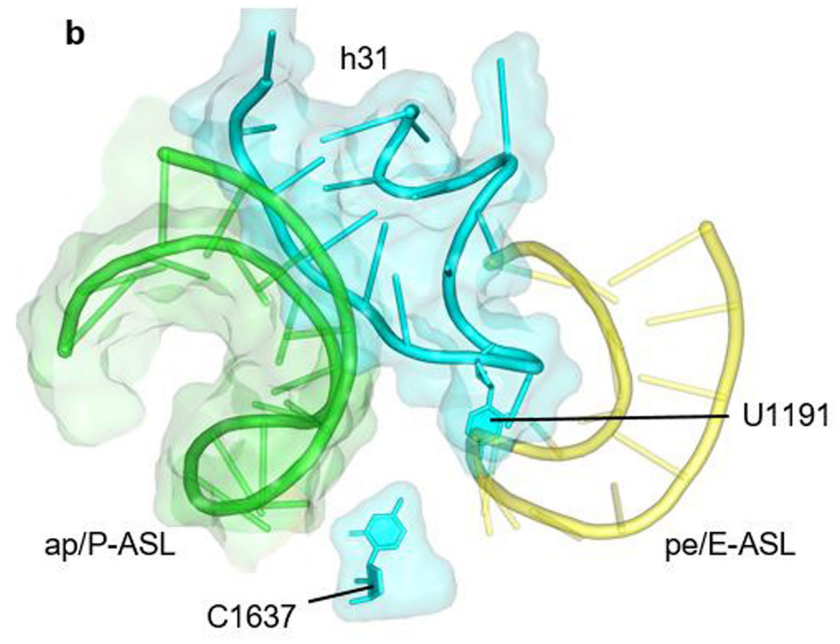

C35 of pe/E tRNA. A similar situation was described for the bacterial ribosome with the pe/E tRNA in a structure modelling spontaneous translocation without $\mathrm{EF}-\mathrm{G}^{37}$. Compared to the intermediate eukaryotic state reported here, the bacterial ap/P tRNA coupled to mRNA is shifted towards the P-site indicating translational reading frame slippage in the factor-free system. This comparison shows the importance of role that translocase eEF2 (or EF-G) fulfils in the coordinated movement of tRNA-mRNA during translocation. 
a
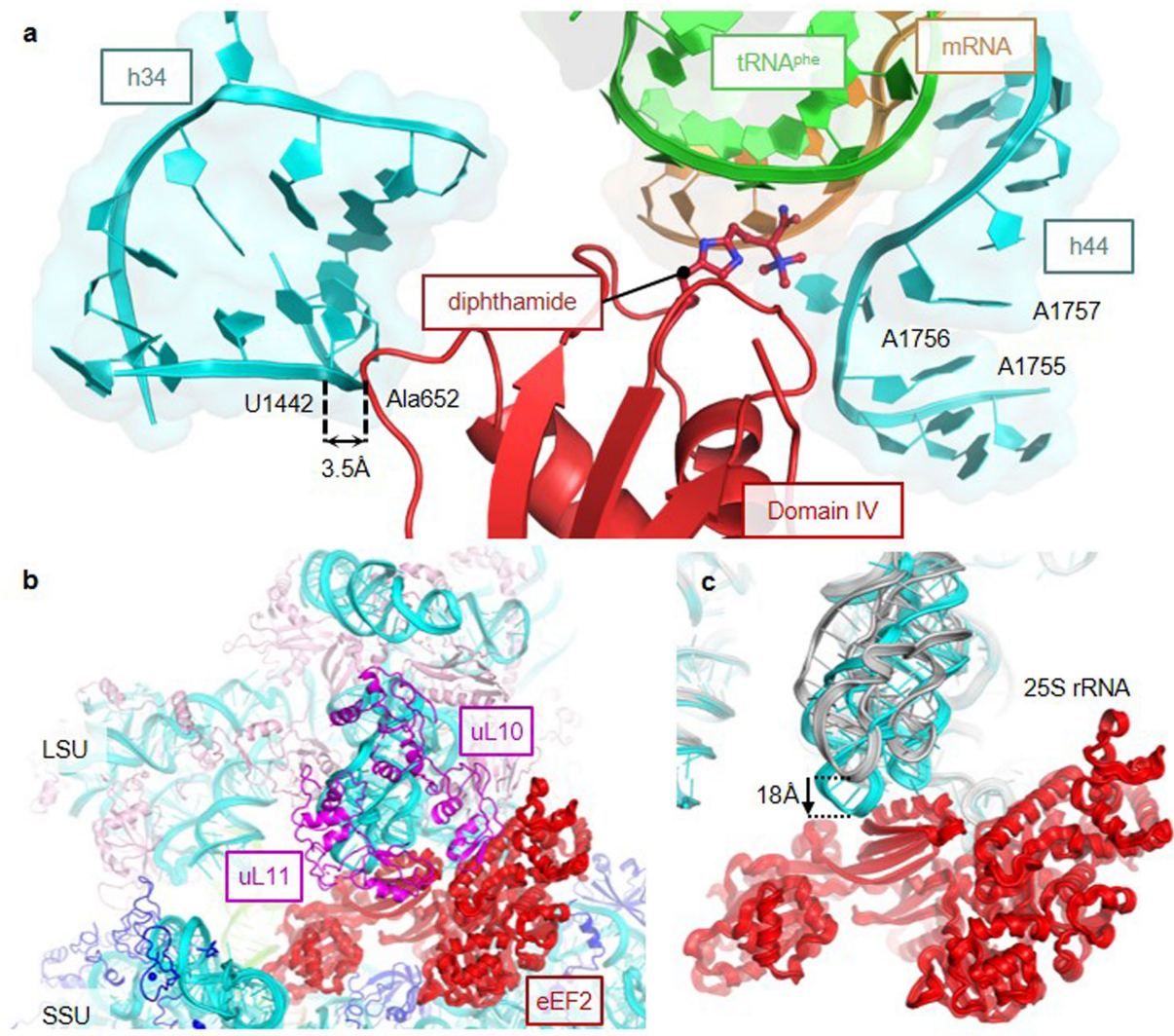

d
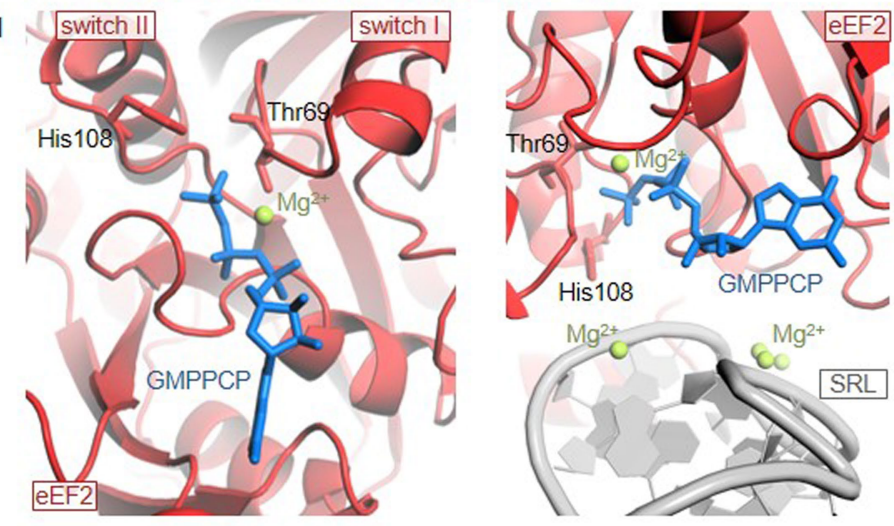

e
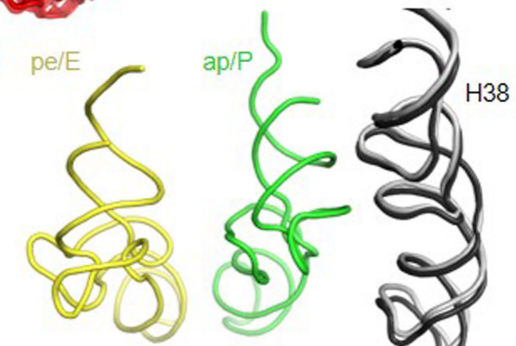

,
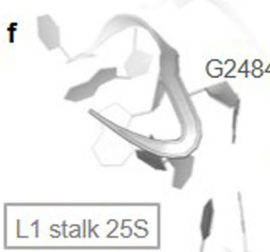
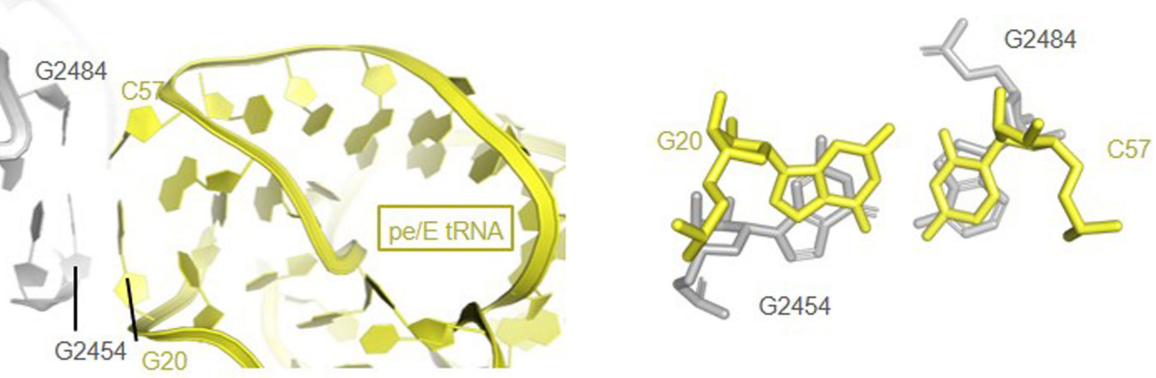

Extended Data Fig. 6 | Contacts between eEF2, tRNAs and the 80 S ribosome in the crystal structure of the translocation intermediate complex. a, Position of diphthamide at the conjunction of ap/P tRNA ${ }^{\text {Phe }}$, mRNA and decoding adenines of $\mathrm{h} 44$ and interactions of eEF2 (Ala652) with rRNA of the SSU head (U1442 of 18S). b, A solvent view on the P stalk region. c, Movement of the $P$ stalk upon eEF2 binding seen when comparing our structure to that of the vacant 80 S ribosome structure (PDB ID 4V88). Alignment was performed using
25S rRNA as a reference. $d$, The $G$ domain of eEF2 with ordered switch I and II regions indicating that this is a pre-hydrolysis state (left panel). Close-up view of the GTP pocket and sarcin-ricin loop (SRL) of 25S rRNA (right panel).e, Disruption of the B1a bridge consisting of helix 38 of $25 \mathrm{~S} r R N A$ (A-site finger) and protein uS13 induced by rotation of the SSU head and body. Non-rotated 80S (PDB ID 3J78) is coloured in black.f, Stacking interactions of rRNA elements (magnified in the right panel) of the L1 stalk with the elbow region of pe/E tRNA. 


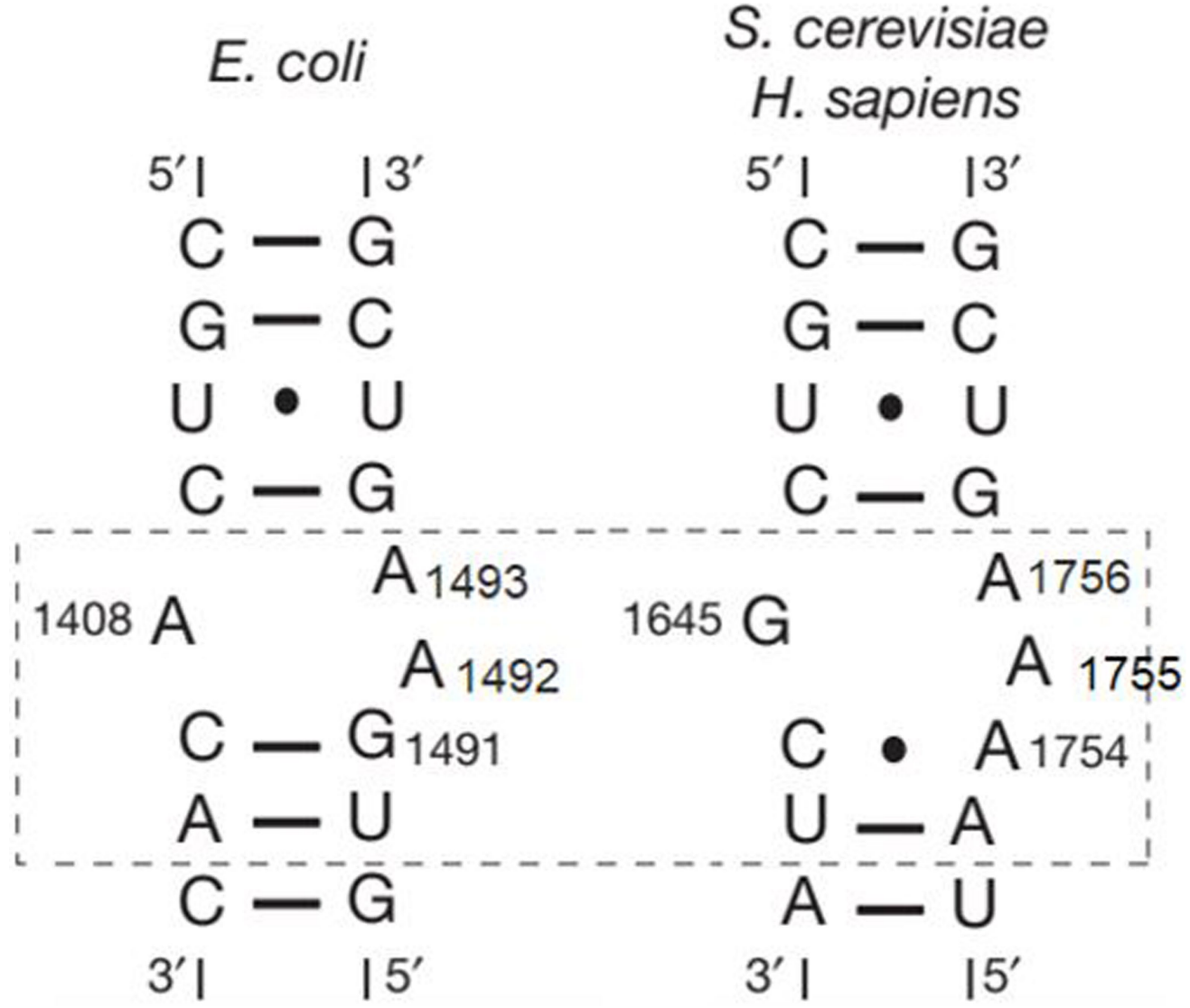

Extended Data Fig. $7 \mid$ Comparison of secondary structures of the decoding loop in bacteria (left) and eukaryotes (right). In bacteria the internal loop of h44 of 16S rRNA consists of two nucleotides (A1492-A1493) on the 3'-side and

contains at least one additional nucleotide on each side is included (dashed box). Secondary structure diagrams of helix 44 from bacteria (16S rRNA, left) and from yeast and human (18S rRNA, right). 
Translocation intermediate complex

\section{Data collection}

Space group

$\mathrm{P} 2{ }_{1} 2{ }_{1} 2_{1}$

Cell dimensions

$$
\begin{aligned}
& a, b, c(\AA) \\
& \alpha, \beta, \gamma\left(^{\circ}\right)
\end{aligned}
$$

Resolution $(\AA)$

$R_{\text {meas }}$

$I / \sigma I$

Completeness (\%)

Redundancy

\section{Refinement}

Resolution $(\AA)$

No. unique reflections

$R_{\text {work }} / R_{\text {free }}$

No. atoms

Protein/RNA

Ligand/ion

$B$-factors

Protein/RNA

Ligand/ion

R.m.s. deviations

Bond lengths $(\AA)$

Bond angles $\left({ }^{\circ}\right)$
233.49299 .85513 .84

909090

$143.9-3.2(3.314-3.2)^{*}$

$0.55(8.34)$

$13.35(0.94)$

99.98 (99.99)

172 (176)

\section{2}

587897

$0.2172 / 0.2780$

206548

578

120.84

87.14

0.005

1.02 


\section{nature portfolio}

Corresponding author(s): Marat Yusupov, Gulnara Yusupova

Last updated by author(s): Oct 4, 2021

\section{Reporting Summary}

Nature Portfolio wishes to improve the reproducibility of the work that we publish. This form provides structure for consistency and transparency in reporting. For further information on Nature Portfolio policies, see our Editorial Policies and the Editorial Policy Checklist.

\section{Statistics}

For all statistical analyses, confirm that the following items are present in the figure legend, table legend, main text, or Methods section.

n/a Confirmed

Х $\square$ The exact sample size $(n)$ for each experimental group/condition, given as a discrete number and unit of measurement

Х $\square$ A statement on whether measurements were taken from distinct samples or whether the same sample was measured repeatedly

$\triangle$ The statistical test(s) used AND whether they are one- or two-sided

Only common tests should be described solely by name; describe more complex techniques in the Methods section.

$\bigotimes \square$ A description of all covariates tested

Х $\square$ A description of any assumptions or corrections, such as tests of normality and adjustment for multiple comparisons

$\triangle \square$ A full description of the statistical parameters including central tendency (e.g. means) or other basic estimates (e.g. regression coefficient)

AND variation (e.g. standard deviation) or associated estimates of uncertainty (e.g. confidence intervals)

For null hypothesis testing, the test statistic (e.g. $F, t, r$ ) with confidence intervals, effect sizes, degrees of freedom and $P$ value noted

$\bigotimes \square$ Give P values as exact values whenever suitable.

$\bigotimes \square$ For Bayesian analysis, information on the choice of priors and Markov chain Monte Carlo settings

$\bigotimes \square$ For hierarchical and complex designs, identification of the appropriate level for tests and full reporting of outcomes

$\bigotimes \square$ Estimates of effect sizes (e.g. Cohen's $d$, Pearson's $r$ ), indicating how they were calculated

Our web collection on statistics for biologists contains articles on many of the points above.

\section{Software and code}

Policy information about availability of computer code

Data collection Crystallographic data were collected at PX1 - X06SA beamline of The Swiss Light Source (SLS) synchrotron at the Paul Scherrer Institut using DA+ data acquisition and analysis software

Data analysis XDS build 20190315

PHENIX 1.14-3260

COOT 0.8 .9

PyMol 2.2.0

For manuscripts utilizing custom algorithms or software that are central to the research but not yet described in published literature, software must be made available to editors and reviewers. We strongly encourage code deposition in a community repository (e.g. GitHub). See the Nature Portfolio guidelines for submitting code \& software for further information.

\section{Data}

Policy information about availability of data

All manuscripts must include a data availability statement. This statement should provide the following information, where applicable:

- Accession codes, unique identifiers, or web links for publicly available datasets

- A description of any restrictions on data availability

- For clinical datasets or third party data, please ensure that the statement adheres to our policy

The atomic coordinates and structure factors for the determined crystal structure have been deposited in the Protein Data Bank with the accession code 70SM (translocation intermediate complex). Previously published models that were used for analysis and comparison are also available from the Protein Data Bank with accession codes 4V88, 4V6F, 1NOU, 3J77, 3J78, 4V6F, 6GZ3, 6GZ5. 


\section{Field-specific reporting}

Please select the one below that is the best fit for your research. If you are not sure, read the appropriate sections before making your selection.

$\bigotimes$ Life sciences $\quad \square$ Behavioural \& social sciences $\quad \square$ Ecological, evolutionary \& environmental sciences

For a reference copy of the document with all sections, see nature.com/documents/nr-reporting-summary-flat.pdf

\section{Life sciences study design}

All studies must disclose on these points even when the disclosure is negative.

Sample size The data set was complete and with a high redundancy as can be seen from the data statistics in Table 1 in accordance with generally accepted crystallographic methods.

Data exclusions No data were excluded from analysis. During data processing heterogeneous crystals which did not scale correctly with the main dataset were removed from further consideration.

Replication All biochemical and crystallization procedures were successfully replicated more than 5 times in independent experiments.

Randomization No randomization was required for the reported experiments, as all variables could be controlled.

Blinding Blinding is not applicable to crystallographic experiments. When needed during analysis of the models we compared our complex structure with previously published native structures.

\section{Reporting for specific materials, systems and methods}

We require information from authors about some types of materials, experimental systems and methods used in many studies. Here, indicate whether each material, system or method listed is relevant to your study. If you are not sure if a list item applies to your research, read the appropriate section before selecting a response.

Materials \& experimental systems

\begin{tabular}{l|l}
\hline n/a & Involved in the study \\
\hline & $\square$ Antibodies \\
\hline & $\square$ Eukaryotic cell lines \\
$\square$ & $\square$ Calaeontology and archaeology \\
$\square$ & $\square$ Clinical data
\end{tabular}

Methods

\begin{tabular}{l|l}
\hline n/a & Involved in the study \\
$\searrow$ & $\square$ ChIP-seq \\
$\searrow$ & $\square$ Flow cytometry \\
X & $\square$ MRI-based neuroimaging
\end{tabular}

